OPEN ACCESS

Edited by:

Quanbao Jiang,

Xi'an Jiaotong University, China

Reviewed by:

Yunhwan Lee,

Ajou University, South Korea

Xiao Zhen $L V$,

Peking University, China

*Correspondence: Hengjin Dong

donghj@zju.edu.cn

Specialty section:

This article was submitted to Aging and Public Health,

a section of the journal

Frontiers in Public Health

Received: 05 August 2021

Accepted: 11 October 2021

Published: 11 November 2021

Citation:

Hu X, Gu S, Zhen X, Sun X, Gu Y and

Dong $H$ (2021) Trends in Cognitive Function Among Chinese Elderly From 1998 to 2018: An Age-Period-Cohort

Front. Public Health 9:753671 doi: 10.3389/fpubh.2021.753671

\section{Trends in Cognitive Function Among Chinese Elderly From 1998 to 2018: An Age-Period-Cohort Analysis}

\author{
Xiaoqian $\mathrm{Hu}^{1,2}$, Shuyan $\mathrm{Gu}^{3}$, Xuemei Zhen ${ }^{4,5}$, Xueshan Sun ${ }^{2}$, Yuxuan $\mathrm{Gu}^{2}$ and \\ Hengjin Dong ${ }^{2,6 *}$ \\ ${ }^{1}$ School of Politics and Public Administration, Qingdao University, Qingdao, China, ${ }^{2}$ Center for Health Policy Studies, School \\ of Public Health, Zhejiang University School of Medicine, Hangzhou, China, ${ }^{3}$ Center for Health Policy and Management \\ Studies, School of Government, Nanjing University, Nanjing, China, ${ }^{4}$ Center for Health Management and Policy, School of \\ Public Health, Shandong University, Jinan, China, ${ }^{5}$ NHC Key Lab of Health Economics and Policy Research, Shandong \\ University, Jinan, China, ${ }^{6}$ The Fourth Affiliated Hospital, Zhejiang University School of Medicine, Yiwu, China
}

Objectives: To investigate the effects of age, period, and cohort (APC) on trends in cognitive function among the Chinese elderly, and to explore how gender gaps in cognitive function change with age, period, and cohort.

Methods: This study used data from the Chinese Longitudinal Healthy Longevity Survey (CLHLS) from 1998 to 2018, and included 90,432 participants aged above 65 years old. The measurement of cognitive function was the score of the Mini-Mental State Examination (MMSE). Cross-classified random-effect models were used to investigate age, period, and cohort trends in cognitive function.

Results: Mini-Mental State Examination scores decreased with age at an increasing rate. While the cohort effect was nearly stable, the period effect demonstrated a downward trend from 1998 to 2002 followed by a nearly flat line. Females were associated with lower MMSE scores than males. When age increased, the gender gaps in MMSE scores further increased. The period-based gender gaps in MMSE scores diverged throughout the 20 years, while the cohort-based gender disparities in MMSE scores converged with successive cohorts.

Conclusions: Age, period, and cohort had different and independent effects on cognitive function among the Chinese elderly. The effect of age was stronger than that of period and cohort. Gender disparities in cognitive function increased with age and period, and decreased with successive cohorts.

Keywords: age-period-cohort, cognition, China, elderly, gender disparity

\section{INTRODUCTION}

Population aging is one of the major challenges worldwide, such as in China (1). In 2020, there were 190.59 million people aged over 65 years old in China, accounting for $13.5 \%$ of the total population (2). In China, life expectancy at birth reached 76.62 years in 2015 (3). The elderly are relatively more vulnerable to cognitive impairment or dementia (4), which would place heavy care burdens on families and societies $(5,6)$. Therefore, the study on health among Chinese elderly, with expanded scales and increased longevity has important significance. 
A better understanding of cognitive function trends is crucial to both the aging population and society because cognitive impairment will decrease the quality of life for the elderly and place care burdens on families of the elderly and social care systems $(5,7)$. Numerous studies have reported that cognitive function improved among the elderly in Sweden $(8,9)$, Denmark (10), Germany (11), the United States (12-14), and China (15). For example, US data suggested that the prevalence of cognitive impairment among the elderly decreased from 1993 to 2004 (14). However, other studies reported opposite trends for cognitive function. For example, findings from a Swedish study indicated that the cognitive function of individuals older than 77 years in 2002 was significantly worse compared with that of individuals interviewed in 1992 (16). Zeng determined that cognitive impairment increased among the Chinese elderly between 1998 and 2008 (1); whereas, these previous studies rarely adjusted for three unique effects related to health trends, which are age, period, and cohort (APC) effects. The effect of age reflects the biological and social processes of aging specific to individuals (17). The effect of period refers to external factors that simultaneously affect all age groups at a particular calendar time. The effect of period often results from shifts in social, technological, historical, and cultural environments, such as technology breakthroughs, world wars, famine, pandemics of infectious diseases, and public health interventions (18). For example, the advent and diffusion of new medical technology, which could prevent the spread of a kind of infectious disease, would reduce mortality rates of all age groups simultaneously (19). This example reflects period effects (20). The effect of cohort reflects different formative life experiences of successive generations $(21,22)$. Cohort effects subsume the effects of early life conditions and continuous exposure to socioeconomic, behavioral, and environmental factors that act persistently over time to produce differences in life course outcomes for specific cohorts (23). One example for the cohort effects would be, for instance, the research of Yang on happiness of Americans from 1972 to 2004 demonstrated that baby boomers experienced less happiness on average than both earlier and later cohorts. Yang explained that this may be closely related to early life conditions and formative experiences. The cohort of baby boomers experienced more competition to enter schools and the labor market because of larger cohort sizes, which might decrease the happiness level of the baby boomers (24). Furthermore, in the past several decades, China has experienced huge societal changes and rapid economic growth, and its people had undergone powerful social forces (15). These societal changes were expected to influence living conditions and the health of populations with distinct effects on different periods and birth cohorts. Since age, period, and cohort had distinct effects on health, these temporal sources of variations in cognitive function need to be distinguished (22).

To examine the cognitive function trends accurately, studies began to explore some of the APC effects which could be improved further. Wu et al. (25) reviewed 70 prevalent studies on dementia in mainland China, Hong Kong, and Taiwan from 1980 to 2012. They identified no significant variation across periods but a potentially increasing cohort effect. However, this study did not conduct complete APC modeling and robust statistical tests because of limited information and considerable variations across different studies. Another study in China reported that the prevalence of cognitive impairment remained stable from 1998 to 2014. Regarding the effect of cohort, cognitive impairment remained stable after a decline in early birth cohorts (25). While this study used the intrinsic estimator method for APC analyses, this method may be a poor approximation of the process of social change (18). Meanwhile, it only focused on the elderly aged 80 years and above, which may provide an incomplete picture of the effects of age. Given these limitations in the literature, studies using advanced methods to explore APC effects on cognitive function trends are required.

Substantial studies had demonstrated that women had worse cognitive function than men in cultural settings such as China (18). For both cultural and historical reasons, females were significantly disadvantaged in nutrition, education, and occupational achievement compared with their male counterparts in traditional China, all of which were associated with cognitive development and maintenance (26). While Chinese females experienced huge transitions and improvement in their living conditions and socioeconomic status across periods and cohorts in this century, the gender gaps in cognitive function may also change. However, few studies explored how gender differentials in cognitive function changed over APC among the Chinese elderly (22). Thus, it is necessary to explicate APC effects on trends of gender gaps in cognitive function, as these effects had been demonstrated in previous studies to be significant contributors to temporal health trends $(19,27)$.

Using data from the Chinese Longitudinal Healthy Longevity Survey (CLHLS), one of the best sources of data on Chinese elderly with multiple birth cohorts from 1998 to 2018, this study intends to investigate the effects of APC on trends of cognitive function among the Chinese elderly and delineate gender disparity trends of cognitive function by APC.

\section{MATERIALS AND METHODS}

\section{Data Sources}

This study used data from the CLHLS, which focused on shedding new light on a better understanding of determinants of healthy longevity. Zeng and Vaupel (28) had introduced the survey design in detail previously. The baseline survey was conducted in 1998; seven follow-up surveys with replacements for deceased samples were conducted in 2000, 2002, 2005, 2008, 2011, 2014, and 2018. These surveys occurred in randomly selected counties and cities in 22 Chinese provinces (28). All centenarians (aged $100+$ years) from these regions who voluntarily agreed to participate in the CLHLS were interviewed. For each centenarian, one nearby octogenarian (aged 80-89 years) and one nearby nonagenarian (aged 9099 years) were matched and interviewed concerning gender and residence. From the 2002 wave, the CLHLS expanded the range of participants to those above 65 years old. Sample weights were made according to the age-gender-residence distribution of the elderly population (28). According to a previous assessment of the CLHLS, the quality of the CLHLS 
data was high regarding data completeness, reliability, and validity (29).

The CLHLS questionnaires comprised questions about basic information, self-reported life evaluation and personality, lifestyle, background, and cognitive function (28). The interview, along with some basic physical examinations, was conducted at the home or nursing institution of each participant by skilled interviewers. Every participant provided written informed consent before the survey. The Research Ethics Committees of Duke University and Peking University granted approval for the protection of human subjects for the CLHLS.

\section{Study Samples}

Considering the CLHLS conducted over 20 years from 1998 to 2018 included the elderly across successive birth cohorts, it was suitable for the exploration of the APC effects on cognitive function trends. There were 92,860 participants aged over 65 years in all eight waves. As the self-reported age after 105 years old was not reliable (28), we excluded 2,202 participants aged over 105 years. Additionally, 226 participants were excluded, as they missed information on cognitive function. Thus, the final sample size in analyses was 90,432.

\section{Variables}

\section{Cognitive Function}

To adapt to Chinese culture, cognitive function in this study was measured by the Chinese version of the Mini Mental State Examination (MMSE), which was modified based on the international standard MMSE questionnaire (30) and tested through pilot survey interviews (28). The MMSE is widely used in clinical and research settings to assess global cognitive function and screen for cognitive impairment (31). The Chinese version of the MMSE included items such as recall, orientation, language, reaction time, and calculation. The participants scored 1 for each correct answer (6). Thus, the range of MMSE score was from 0 to 30 , with relatively high scores indicating better cognitive function.

\section{Age, Period, and Cohort}

For ease of interpretation of the intercept values, the age of the participants divided by 10 was the grand mean-centered (18). Period indicated the year the survey was conducted $(1998,2000$, 2002, 2005, 2008, 2011, 2014, and 2018). Cohort was the year the participants were born. Participants who were born before 1900 or after 1940 were grouped separately to ensure a sufficient number of participants (32). We subsequently grouped other birth cohorts into 5-year bands.

\section{Covariates}

Gender (male $=0$; female $=1$ ) is the key stratification factor to explore age-period-cohort effects on gender disparity in cognitive function. We also adjusted for the samples' demographic characteristics, socioeconomic status (SES), health behaviors, chronic diseases and prior test exposure (representing their associations with cognitive function in previous studies)
(33). Demographic characteristics included ethnicity, current residence, birthplace, marital status, and co-residence. Ethnicity was defined as Han and minority (including all ethnic groups except Han). Current residence and birthplace were both dichotomized as urban and rural. Marital status was defined as married and not married (we combined divorced, separated, widowed, and never married as not married). Co-residence was defined as alone and living with others (such as household members and living in a nursing home). SES included education and job. Education was defined as illiterate (had not received any education) and literate. The CLHLS collected job information of the participants through the following question: "What was your main occupation before age 60?" Considering most people were farmers at that time, we defined job as farmers and others (such as professional and technical personnel; industrial worker; governmental, institutional, or managerial personnel; commercial or service worker; military personnel, and others). Health behaviors indicating the lifestyle of the participants at survey time included smoking, drinking, and physical exercise, which were dichotomized as yes and no. Chronic diseases included four common diseases among the elderly, hypertension, diabetes, heart disease, and stroke. Prior test exposure was used to adjust for potential practice effects of repeat cognitive function testing (14). Participants who had participated in previous waves of the survey was defined as "yes," otherwise was defined as "no."

\section{Statistical Methods}

First, we summarized the basic characteristics of study samples in all the eight waves using means \pm standard deviation or frequency (percentages).

We applied hierarchical APC (HAPC) models to simultaneously estimate the age, period, and cohort trends of the MMSE score for repeated cross-sectional data. The HAPC model was developed by Yang and her colleague, in which the three effects are not assumed to be additive at the same level of analysis (18). The HAPC model could address a classical APC identification problem in two ways (18). First, we grouped individuals born in a 5-year range into a single cohort to break the linear dependence among the dimensions of APC. Second, the nonlinear transformations approach suggested applying a parametric nonlinear transformation, such as polynomials, to at least one of the APC dimensions to break their linear relationships (24). According to this strategy and previous findings on curvilinear age effects on health (18), this study proposed models of MMSE scores as a quadratic function of age.

Thus, we fit HAPC cross-classified random effect regression models (HAPC-CCREMs) to examine the effects of APC on cognitive function. In each regression model, MMSE scores were regressed on age in linear and squared terms and other confounding variables as required. The coefficients of period, cohort, and gender were allowed to have random effects (24). This design made it possible to explore the period-based and cohort-based trends of gender gaps in cognitive function. In total, the model took the following form: 
TABLE 1 | Basic characteristics of samples in the eight surveys.

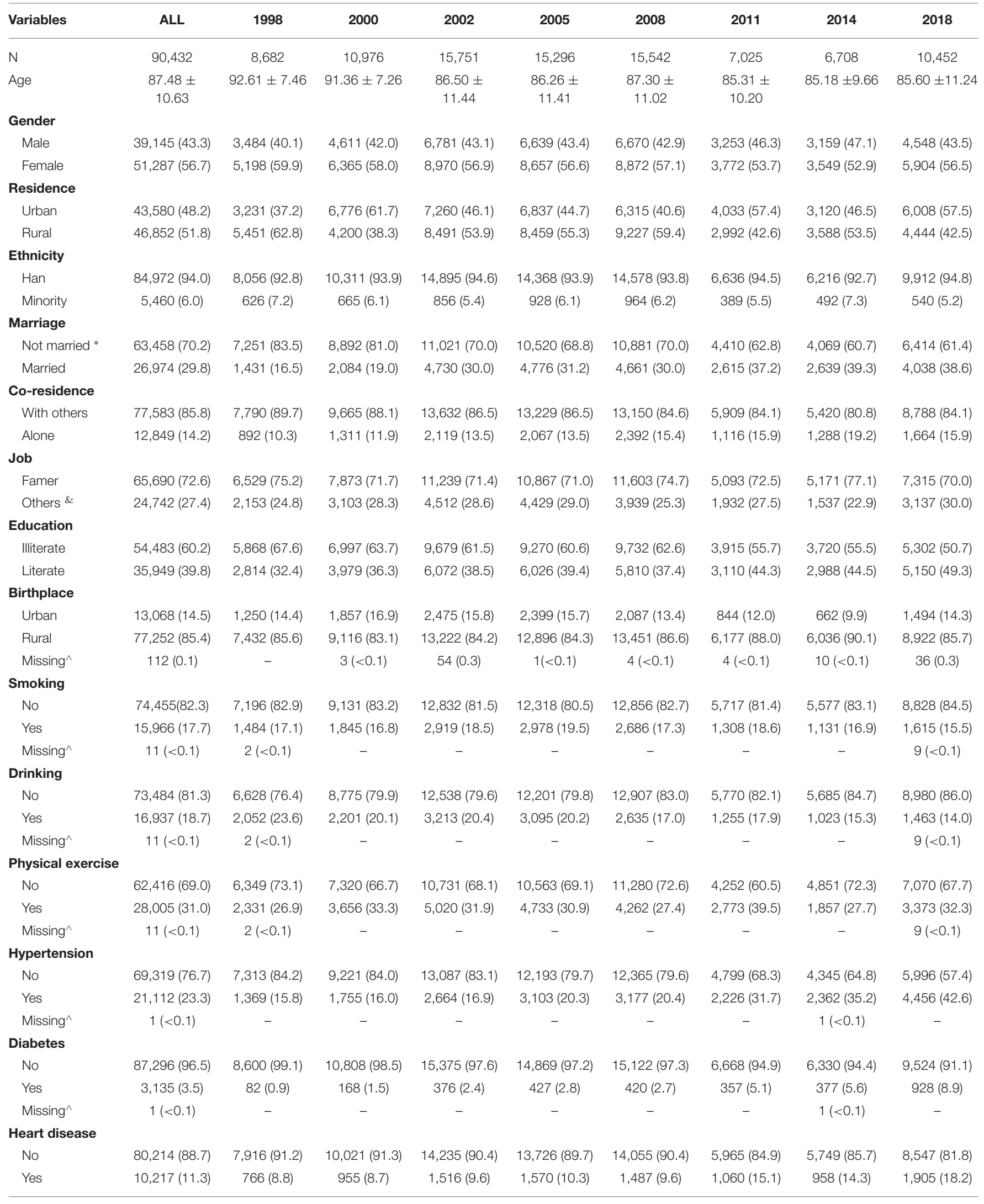


TABLE 1 | Continued

\begin{tabular}{|c|c|c|c|c|c|c|c|c|c|}
\hline Variables & ALL & 1998 & 2000 & 2002 & 2005 & 2008 & 2011 & 2014 & 2018 \\
\hline Missing^ & $1(<0.1)$ & - & - & - & - & - & - & $1(<0.1)$ & - \\
\hline \multicolumn{10}{|l|}{ Stroke } \\
\hline No & 84,354 (93.3) & 8,345 (96.1) & 10,485 (95.5) & 14,848 (94.3) & 14,369 (93.9) & $14,572(93.8)$ & 6,391 (91.0) & $6,071(90.5)$ & $9,273(88.7)$ \\
\hline Yes & $6,077(6.7)$ & 337 (3.9) & $491(4.5)$ & $903(5.7)$ & $927(6.1)$ & $970(6.2)$ & $634(9.0)$ & $636(9.5)$ & $1,179(11.3)$ \\
\hline Missing ${ }^{\wedge}$ & $1(<0.1)$ & - & - & - & - & - & - & $1(<0.1)$ & - \\
\hline \multicolumn{10}{|c|}{ Prior test exposure } \\
\hline No & $51,099(56.5)$ & $8,682(100.0)$ & $6,448(58.7)$ & 9,651 (61.3) & 7,387 (48.3) & 8,313 (53.5) & $47(0.7)$ & 2,085 (31.1) & 8,486 (81.2) \\
\hline Yes & $39,333(43.5)$ & $0(0.0)$ & 4,528 (41.3) & $6,100(38.7)$ & 7,909 (51.7) & 7,229 (46.5) & 6,978 (99.3) & 4,623 (68.9) & 1,966 (18.8) \\
\hline MMSE score & $21.80 \pm 9.23$ & $21.15 \pm 8.96$ & $21.22 \pm 9.11$ & $22.02 \pm 8.80$ & $22.07 \pm 9.31$ & $\begin{array}{c}20.79 \pm \\
10.03\end{array}$ & $22.60 \pm 8.84$ & $23.12 \pm 8.71$ & $22.37 \pm 9.22$ \\
\hline
\end{tabular}

MMSE, Mini Mental State Examination. Data are presented as mean \pm standard deviation or $n(\%)$.

$\wedge$ Missing data were excluded from other percentage calculation.

* This category included divorced, separated, widowed, and never married.

\& This category included professional and technical personnel; industrial worker; governmental, institutional or managerial personnel; commercial or service worker; military personnel, and others.

Level 1 model:

$$
\begin{aligned}
M M S E_{i j k} & =\beta_{0 j k}+\beta_{1} A_{i j k}+\beta_{2} A_{i j k}^{2}+\beta_{3 j k} S_{i j k}+\sum_{p=4}^{P} \beta_{p} X_{p i j k} \\
& +e_{i j k}, e_{i j k} \sim N\left(0, \sigma^{2}\right)
\end{aligned}
$$

where $M M S E_{i j k}$ stands for scores of MMSE for respondent $i$ (for $i=1,2, \ldots, n_{j k}$ ) within period $j$ (for $j=1,2, \ldots, 7$ ) and cohort $k$ (for $k=1,2, \ldots, 10$ ); $A$ and $A^{2}$ denote age and agesquared, respectively; $S$ denotes gender; $X_{p}$ denotes the vector of other individual-level variables, such as age by gender, to test how the gender gap in cognitive function varies from age and covariates. $\beta_{0 j k}$ is the intercept indicating the cell mean for the reference group at the mean age interviewed in period $j$ and belonging to cohort $k ; \beta_{1}$ and $\beta_{2}$ denote the fixed coefficients for age; $\beta_{3 j k}$ denotes the random coefficients for gender; $\beta_{p}$ denotes the fixed coefficients for covariates; $P$ is the maximum number of covariates included; $e_{i j k}$ is the random individual effect or cell residual, which is assumed to be normally distributed with mean 0 and a within-cell variance $\sigma^{2}$. Age divided by 10 is the grand mean-centered for ease of interpretation of the intercept values.

Level 2 model:

$$
\begin{aligned}
& \beta_{0 j k}=\gamma_{0}+u_{0 j}+v_{0 k} \\
& \beta_{3 j k}=\gamma_{3}+u_{3 j}+v_{3 k}
\end{aligned}
$$

The level 2 models test whether with gender disparities in MMSE scores or not, varied by period or cohort through the specifications of random variance components for the random intercept and coefficients. $\beta_{0 j k}$ denotes a random intercept, which specifies that the overall mean varies from period to period and from cohort to cohort. $\gamma_{0}$ is the expected mean at zero values of all level 1 variables averaged over all periods and cohorts; $u_{0 j}$ is the overall period effect regarding residual random coefficients of period $j$ averaged over all cohorts with variance $\sigma_{u 0} ; v_{0 k}$ is the overall cohort effect regarding residual random coefficients of cohort $k$ averaged over all periods with variance $\sigma_{v 0} \cdot \beta_{3 j k}$ denotes the random coefficients for gender; $\gamma_{3}$ is the level 2 fixed-effect coefficient that represents the fixed effects of gender. To test whether the gender stratifications of MMSE scores varied by period or cohort, we specify that coefficients have period effects $\left(u_{3 j}\right)$ and cohort effects $\left(v_{3 k}\right)$ whose corresponding random variance components are $\sigma_{u 3}$ and $\sigma_{v 3}$. These random variance components of period and cohort for the intercept and coefficients are assumed to have multivariate normal distributions (24).

Therefore, in the level 1 model, we could test whether the gender disparity in MMSE scores varied with age by the interaction term of age with gender. The level 2 model could test whether this gap varied by period or cohort. Based on the combination of two-level models, we used six models to explore the effects of APC on trends of MMSE scores and change in gender disparities in MMSE scores with APC. Model 1 was a two-level model with a fixed effect for age and random effects for period and cohort to explore the net effects of APC on MMSE scores. Model 2 added the key independent variable, gender, to explore its influence on MMSE scores. Model 3 added the interaction between age and gender to explore how the gender disparity in MMSE scores varied with age. Model 4 adjusted confounding variables based on Model 3. Model 5 added random effects of coefficients of gender to explore how gender disparities in MMSE scores varied by period and cohort. Model 6 added covariates based on Model 5 to use a full model. Analyses were conducted using SAS PROC MIXED (18). Bayesian Information Criterion (BIC) was used to compare models concerning the goodness of fit, with a smaller BIC value indicating better model fit (34).

\section{RESULTS}

\section{Basic Characteristics of Samples}

Table 1 presents basic characteristics of the samples in the eight surveys from 1998 to 2018. In total, there were 90,432 
TABLE 2 | Hierarchical age-period-cohort cross-classified random-effect model estimates of MMSE scores.

\begin{tabular}{|c|c|c|c|c|c|c|c|c|c|c|c|c|}
\hline & \multicolumn{2}{|c|}{ Model 1} & \multicolumn{2}{|c|}{ Model 2} & \multicolumn{2}{|c|}{ Model 3} & \multicolumn{2}{|c|}{ Model 4} & \multicolumn{2}{|c|}{ Model 5} & \multicolumn{2}{|c|}{ Model 6} \\
\hline & Coef. & SE & Coef. & SE & Coef. & SE & Coef. & SE & Coef. & SE & Coef. & SE \\
\hline \multicolumn{13}{|l|}{ Fixed effects } \\
\hline Intercept & $22.899^{\star \star \star}$ & 0.225 & $24.311^{\star \star \star}$ & 0.262 & $24.368^{\star \star \star}$ & 0.263 & $22.433^{\star \star \star}$ & 0.264 & $24.337^{\star \star \star}$ & 0.269 & $22.413^{\star \star \star}$ & 0.251 \\
\hline Age & $-4.585^{\star \star \star}$ & 0.086 & $-4.597^{\star \star \star}$ & 0.064 & $-4.018^{* \star \star}$ & 0.074 & $-3.462^{\star \star \star}$ & 0.070 & $-4.165^{\star \star \star}$ & 0.070 & $-3.449^{\star \star \star}$ & 0.067 \\
\hline $\mathrm{Age}^{2}$ & $-0.937^{\star * *}$ & 0.037 & $-1.015^{\star \star *}$ & 0.032 & $-0.944^{\star \star \star}$ & 0.033 & $-0.942^{\star \star \star \star}$ & 0.032 & $-0.958^{\star \star \star}$ & 0.033 & $-0.931^{\star \star \star}$ & 0.032 \\
\hline Gender (female = 1) & & & $-2.159^{\star \star \star}$ & 0.053 & $-2.241^{\star \star \star}$ & 0.054 & $-0.868^{\star \star \star}$ & 0.064 & $-2.107^{\star \star}$ & 0.446 & $-0.872^{\star \star \star}$ & 0.114 \\
\hline Age * Gender & & & & & $-0.917^{\star \star \star}$ & 0.052 & $-0.880^{\star \star \star}$ & 0.051 & & & $-0.892^{\star \star \star}$ & 0.055 \\
\hline Residence (rural = 1) & & & & & & & $-0.174^{\star \star}$ & 0.057 & & & $-0.170^{* \star}$ & 0.057 \\
\hline Ethnic (minority = 1) & & & & & & & $0.969^{* \star *}$ & 0.106 & & & 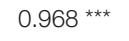 & 0.106 \\
\hline Marriage $($ married $=1$ ) & & & & & & & $0.971^{\star \star \star *}$ & 0.070 & & & $0.974^{* * \star}$ & 0.070 \\
\hline Living condition (alone = 1) & & & & & & & $1.403^{* \star *}$ & 0.076 & & & $1.403^{* \star \star}$ & 0.076 \\
\hline Job (farmer = 1) & & & & & & & $-0.266^{\star \star \star}$ & 0.070 & & & $-0.271^{\star \star \star}$ & 0.070 \\
\hline Education (literate = 1) & & & & & & & $1.611^{\star \star \star}$ & 0.064 & & & $1.615^{\star \star \star}$ & 0.064 \\
\hline Birthplace (rural = 1) & & & & & & & $-0.589^{\star \star \star}$ & 0.079 & & & $-0.584^{\star * \star}$ & 0.079 \\
\hline Smoking (yes = 1) & & & & & & & $0.234^{\star \star}$ & 0.073 & & & $0.233^{\star \star}$ & 0.073 \\
\hline Drinking (yes = 1) & & & & & & & $0.785^{\star \star \star}$ & 0.069 & & & $0.785^{\star \star \star}$ & 0.069 \\
\hline Physical exercise (yes = 1) & & & & & & & $2.504^{\star \star \star}$ & 0.058 & & & $2.508^{\star \star \star}$ & 0.058 \\
\hline Hypertension (yes = 1) & & & & & & & $0.257^{\star \star \star}$ & 0.064 & & & $0.259^{* * *}$ & 0.064 \\
\hline Diabetes $($ yes $=1)$ & & & & & & & -0.261 & 0.142 & & & -0.260 & 0.142 \\
\hline Heart disease (yes = 1) & & & & & & & 0.018 & 0.083 & & & 0.020 & 0.083 \\
\hline Stroke $($ yes = 1) & & & & & & & $-2.976^{\star \star \star}$ & 0.103 & & & $-2.980^{\star \star \star}$ & 0.103 \\
\hline Prior test exposure (yes $=1$ ) & & & & & & & $-0.185^{\star \star}$ & 0.060 & & & $-0.187^{\star *}$ & 0.060 \\
\hline \multicolumn{13}{|l|}{ Variance components } \\
\hline \multicolumn{13}{|l|}{ Period } \\
\hline Intercept & $0.355^{*}$ & 0.194 & $0.453^{*}$ & 0.248 & 0.451 * & 0.248 & $0.380^{\star}$ & 0.210 & $0.482 *$ & 0.272 & 0.340 * & 0.191 \\
\hline Gender & & & & & & & & & 0.314 * & 0.185 & 0.062 & 0.043 \\
\hline \multicolumn{13}{|l|}{ Cohort } \\
\hline Intercept & 0.039 & 0.028 & $0.087^{*}$ & 0.051 & $0.098^{*}$ & 0.056 & 0.074 * & 0.043 & 0.089 & 0.072 & 0.062 & 0.038 \\
\hline Gender & & & & & & & & & $1.563 *$ & 0.757 & 0.007 & 0.015 \\
\hline \multicolumn{13}{|l|}{ Model fit } \\
\hline $\mathrm{BIC}$ & \multicolumn{2}{|c|}{$708,261.5$} & \multicolumn{2}{|c|}{$628,065.5$} & \multicolumn{2}{|c|}{$627,755.3$} & \multicolumn{2}{|c|}{$622,020.3$} & \multicolumn{2}{|c|}{$627,809.3$} & \multicolumn{2}{|c|}{$621,999.8$} \\
\hline
\end{tabular}

MMSE, Mini Mental State Examination; SE, standard error; Coef., coefficient; BIC, Bayesian Information Criterion.

${ }^{\star} p \leq 0.05 ;{ }^{* *} p \leq 0.01 ;{ }^{* \star *} p \leq 0.001$.

participants with an average age of 87.48 years old. Most of the respondents were Han, living with others, born in a rural area, illiterate, working as farmers, and not married. About $80 \%$ of the participants did not smoke and drink. The average MMSE score of all the samples was 21.8 and ranged from 20.79 to 23.12 among the eight surveys.

\section{Age-Period-Cohort Trends and Differentials in MMSE Scores}

Table 2 presents estimates of fixed effects of all individuallevel covariates and random-effect variance components. Model 1 showed that with only APC effects included in the model, the predicted average overall MMSE score was 22.9. Age had a significant negative quadratic effect on MMSE scores (coef. for age $=-4.585, p<0.001$; coef. for age $\mathrm{a}^{2}=-0.937, p<$ 0.001 ), which suggested that after period and cohort effects were taken into consideration, MMSE scores declined at an accelerated rate with age. Level 2 results suggested that MMSE scores varied in a smaller magnitude by period and cohort (coef. for period $=0.355, p=0.034$; coef. for cohort $=0.039$, $p=0.081$ ), relative to the effect of age. Figure 1 presents the overall trends of cognitive function in terms of predicted MMSE scores, estimated from model 1. Figure 1A showed curvilinear age effects. Figure 1B shows the effect of estimated period, which was calculated as $\hat{\beta}_{0 j}=\hat{\gamma}_{0}+u_{0 j}$, where $\hat{\gamma}_{0}$ was the intercept or estimated overall mean and $u_{0 j}$ was the period-specific random-effect coefficients estimated from model 1. The effect of period demonstrated a downward trend from 1998 to 2002, followed by a nearly flat line. Figure 1C displays the estimated cohort effects in terms of the predicted MMSE scores at the mean age and averaged over all periods. Similar to the effect of period, the effect of cohort effect was calculated as $\hat{\beta}_{0 k}=\hat{\gamma}_{0}+v_{0 k}$, where $v_{0 k}$ was the cohortspecific random-effect coefficients estimated from model 1 . The 
A

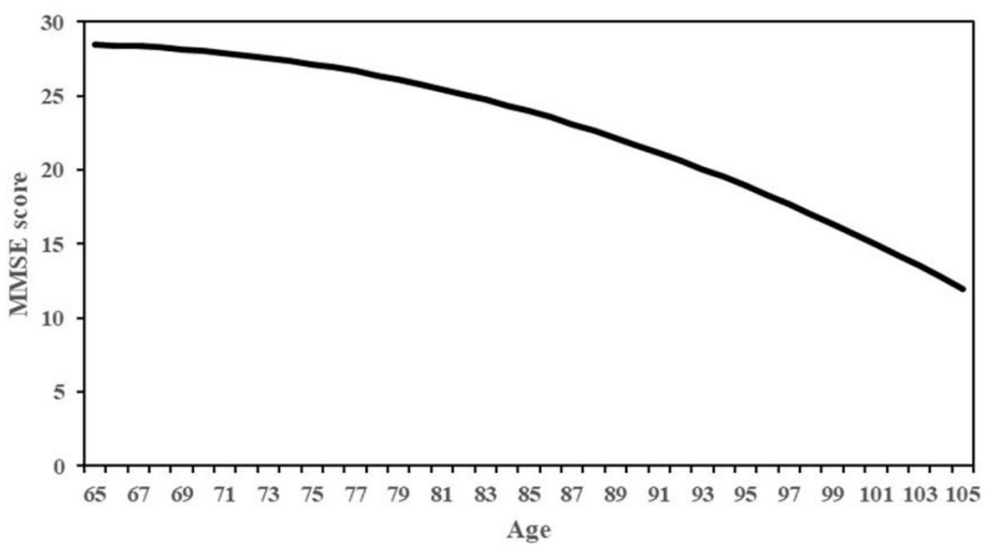

B

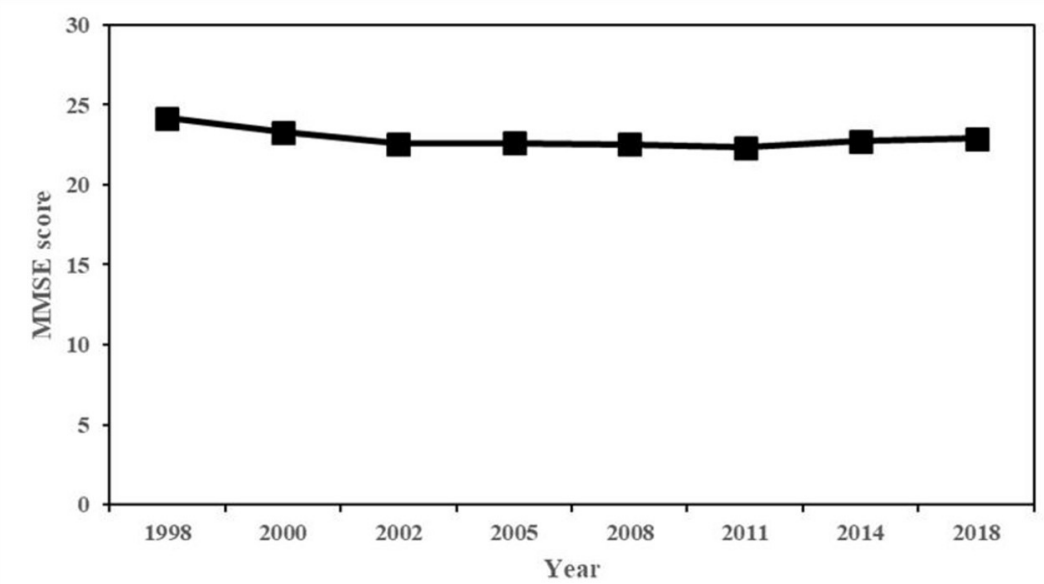

C

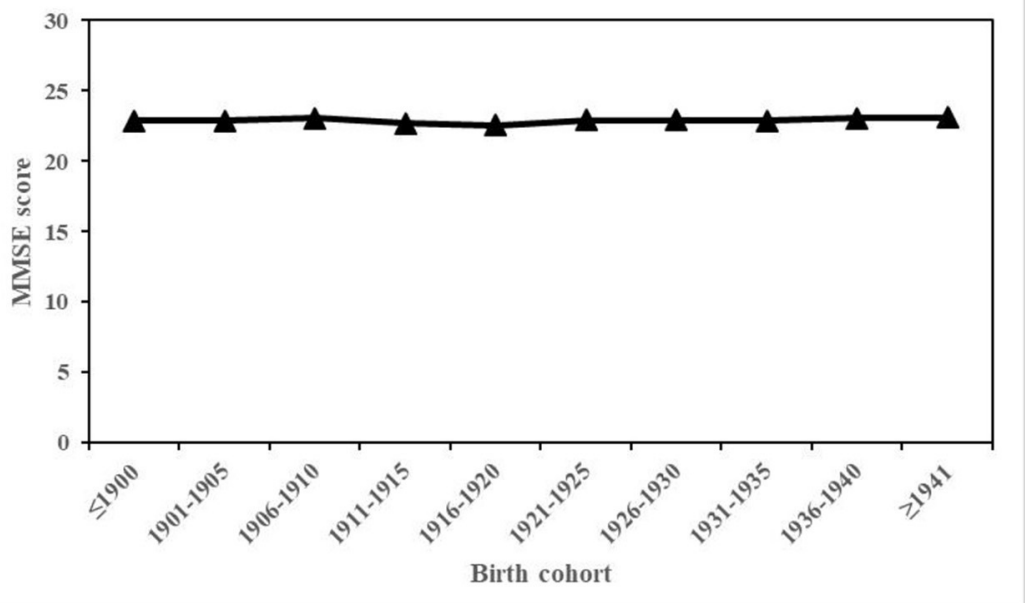

FIGURE 1 | Overall age, period, and cohort effects on MMSE scores. (A) Age. (B) Period. (C) Cohort. 
effect of cohort was not significant, demonstrating a trend with little change.

\section{Age-Period-Cohort Trends of Gender Disparities in MMSE Scores}

Model 2 indicated that the females had significantly lower MMSE scores (coef. $=-2.159, p<0.001$ ) relative to the males when the effects of APC were considered. Model 3 indicated that the gender disparity in MMSE scores varied significantly with age (coef. $=-0.917, p<0.001$ ). When age increased, the gender gap further increased (Figure 2A). Model 4 revealed that rural residence, ethnicity, marital status, living condition, job, education, birthplace, smoking, drinking, physical exercise, hypertension, stroke, and prior test exposure had significant influences on MMSE scores. Those participants who were born in a rural area, illiterate, farmers, not in a marriage, living with others, and suffering from stroke had lower MMSE scores. Comparing model 3 with model 4 , the interaction effect of gender with age remained highly significant but decreased a little in size when confounding variables were considered.

When it came to the effects of period on gender disparities in MMSE scores, model 5 demonstrated that the gender gap in MMSE scores varied significantly with period net of age and cohort (coef. $=0.314, p=0.046$ ). Figure 2B displays the estimated random period effects on gender disparities in MMSE scores, which was calculated as $\hat{\beta}_{0 j}+\hat{\beta}_{3 j}=\hat{\gamma}_{0}+\hat{\gamma}_{3}+u_{0 j}+$ $u_{3 j}$ (where $\hat{\gamma}_{0}$ was the intercept or estimated overall mean, $\hat{\gamma}_{3}$ was the estimated fixed gender effect coefficient, $u_{0 j}$ was the period-specific random-effect coefficients, and $u_{3 j}$ was the gender-specific random period effects). Figure 2B shows that the gap in MMSE scores between the males and females further increased throughout the 20 years. Although the MMSE scores for both genders indicated decreasing trends, those of the females declined more.

Cohort effects on the gender disparity in MMSE scores were also significant when age and period were considered (coef. $=$ 1.563, $p=0.019$ ) (model 5 in Table 2). Figure 2C displays the estimated random cohort effect on gender disparities in MMSE scores, which was calculated as $\hat{\beta}_{0 k}+\hat{\beta}_{3 k}=\hat{\gamma}_{0}+\hat{\gamma}_{3}+v_{0 k}+v_{3 k}$ Similar to the period effect, $v_{0 k}$ was the cohort-specific randomeffect coefficient and $v_{3 k}$ was the gender-specific random cohort effect. From Figure 2C, we identified that the gap in MMSE scores between genders decreased across cohorts, which was largely because of the increasing trend of MMSE scores for females and the relatively stable trend for males among successive cohorts. Model 6, the final model, showed that period and cohort effects on gender differentials in MMSE scores were not statistically significant when covariates were taken into account.

\section{DISCUSSION}

Using eight waves of the CLHLS data from 1998 to 2018, we applied HAPC-CCREMs to explore trends of cognitive function among the Chinese elderly. Our findings indicated that the MMSE scores decreased with age at an accelerated rate. While the effect of cohort was nearly stable, the effect of period demonstrated a downward trend from 1998 to 2002 followed by a nearly flat line. The females were associated with lower MMSE scores than males. When age increased, the gender gap in cognitive function further increased. The period trends of gender gaps in MMSE scores widened throughout the 20 years, while the cohort trends of gender disparities in MMSE scores narrowed with successive cohorts.

The results of the APC model analysis indicated that the effect of APC on cognitive function among the Chinese elderly were distinct and independent of each other. These different effects suggested that it is of vital importance to test variations formally in all three time-related dimensions in studies on trends in health (24). In line with previous studies (22), we identified that the MMSE scores decreased with age at an accelerated rate. Compared with the effect age, the effect of period and cohort was smaller. The effect of period demonstrated a downward trend from 1998 to 2002, followed by a nearly flat line. The downward trend may partly be because of in 1998 and 2000 waves of the survey, CLHLS mainly focused on elders above 80 years old. Those oldest-olds who could survive to advanced ages usually had relatively better health status (35), resulting in higher MMSE scores in the 1998 and 2000 waves than scores in the follow-up waves of survey including elders above 65 years old. The stable period trend from 2002 to 2018 was consistent with previous research (22). Zhang also determined that with the net of age and cohort effect, the period-based trend among the Chinese elderly was relatively stable (22). The effect of cohort was not significant, demonstrating a trend with little change from our results, which were different from another Chinese study (22). According to the results of Zhang, cognitive impairment declined across birth cohorts. Zhang used the rate of cognitive impairment as the dependent variable, while our studies used scores of cognitive function, including both the rate and severity of cognitive impairment, which may have different results.

We identified that the females had significantly worse cognitive function than the males, consistent with the results from Taiwan (36) and India (37) but different from the results from developed countries $(31,38)$. This may be due to the long-lasting preference for sons in traditional Chinese society; compared with males, most females had a relatively tough early life with bad nutrition (33) and few opportunities to obtain education (39), all of which were associated with disadvantages in cognitive development and maintenance (26). Furthermore, this gender disparity in cognitive function further increased with increase in age from our results, supporting the cumulative disadvantage theory (40). According to the cumulative disadvantage theory, early disadvantages would accumulate over the life course by setting people onto different life trajectories, resulting in increasing disparities in health as people age (33). Considering the bad cognitive function and longevity of females, more attention and resources should be given to them to ensure they obtain adequate care.

Gender disparities in MMSE scores enlarged significantly from 1998 to 2018. Although the MMSE scores for both genders indicated decreasing trends, those of the females declined more. Similarly, Zeng determined that the elderly had lower MMSE scores than those of the same age interviewed 10 years ago; 
A

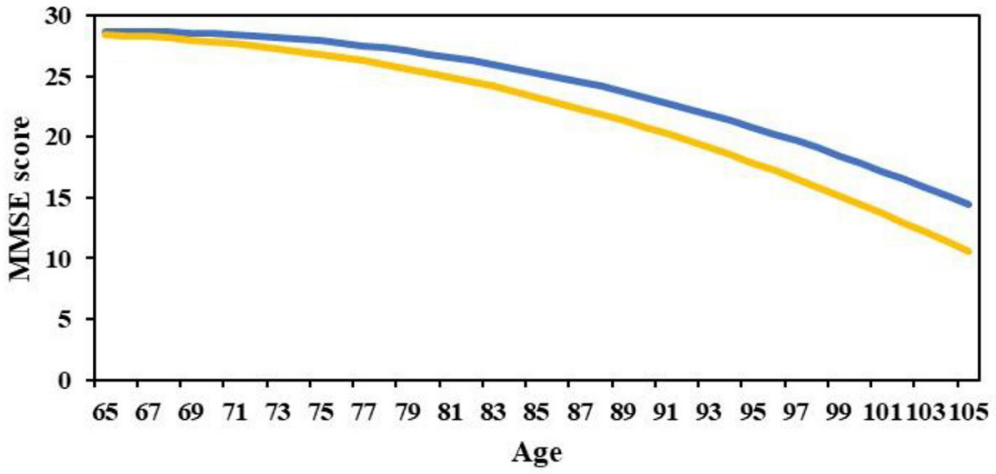

male female

B

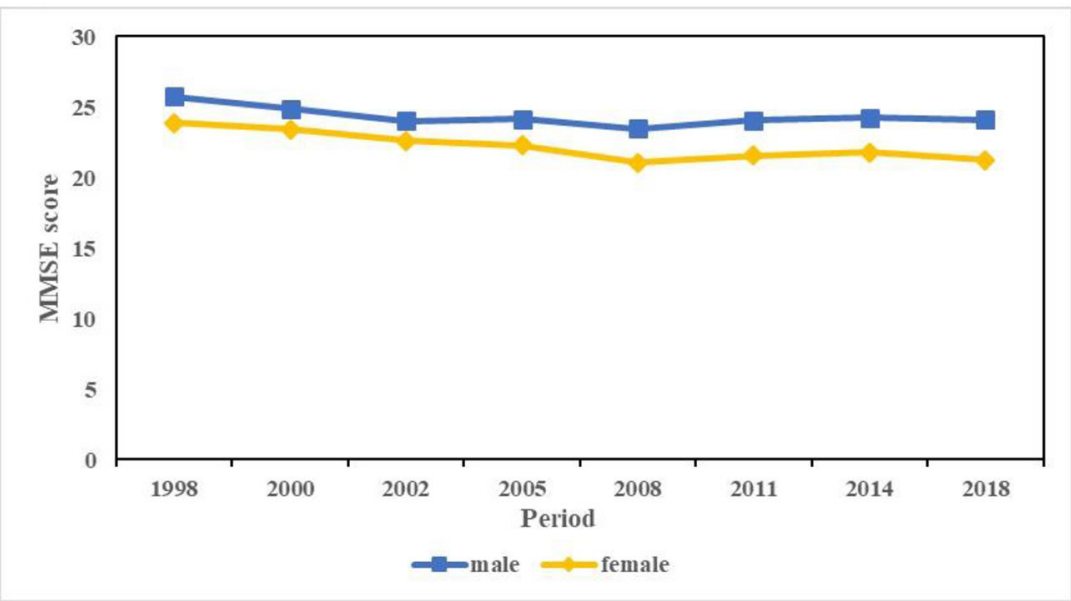

C

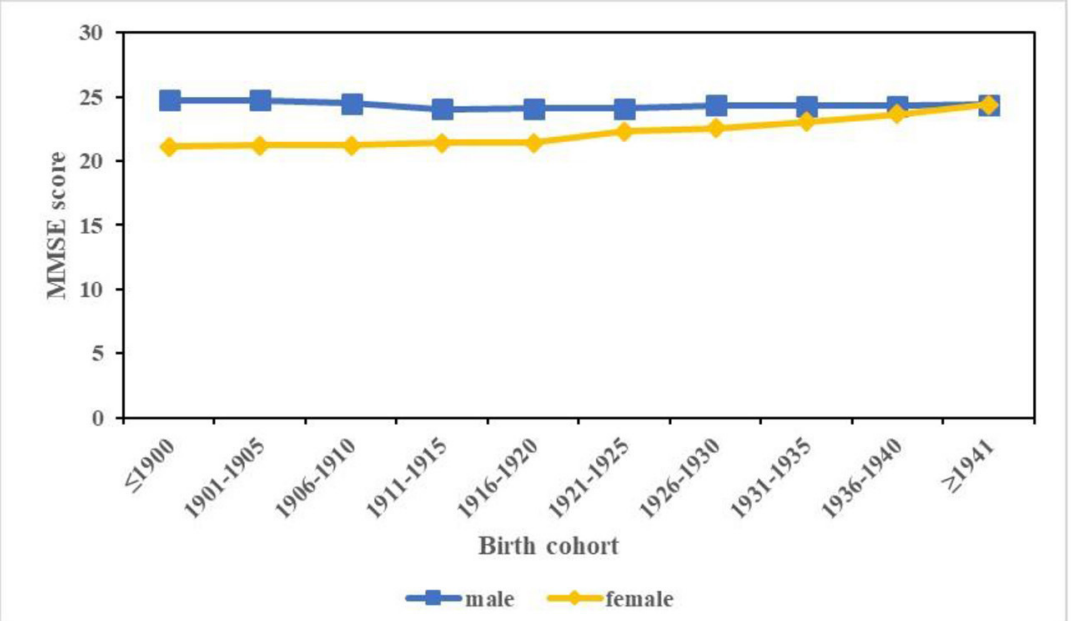

FIGURE 2 | Predicted age, period, and cohort trends in the gender disparity in MMSE scores. (A) Age. (B) Period. (C) Cohort. 
meanwhile, the cognitive function of females declined faster than that of males (1). This finding could be explained by the expansion of the morbidity theory, indicating that improvements in medical conditions and living standards in recent years may result in some frail elderly individuals being saved from dying but surviving with poor cognitive function, which may reduce the whole scores of cognitive function (10).

We observed that the cohort trends of gender disparities in cognitive function narrowed with cohorts, which may largely be because of the increase in MMSE scores for females among successive cohorts. The improvement in cognitive function for females in late-born cohorts that we found was consistent with previous studies $(22,41)$. We speculated that the decreasing gender gap in cognitive function with cohort could be substantially explained by differential exposures to various social correlates of cognitive function, especially the access of women to schooling had improved significantly over time in China (42). Numerous studies from different countries had demonstrated the association between education and late-life cognition as measured by cognitive tests, cognitive impairment, or dementia (33). Education would not only promote cognitive development in early life, but also lead to higher SES, better living condition, and more cognitive reserve in later life $(26,43)$. Thus, public policy targeting education is required, which will not only improve the lives of children but also enhance cognitive well-being and bridge the gender gaps of the elderly ultimately.

While our results provided new insights into the trends of cognitive function among the Chinese elderly, there were some limitations. First, we performed MMSE to evaluate cognitive function rather than comprehensive clinical evaluations. MMSE is a screening tool and provides global cognitive function. The clinical evaluations are more accurate; hence further analyses by different domains of cognitive function are needed. Second, because of data limitation, the earliest and latest birth cohorts did not capture a full age distribution, which may bias the estimates for cohort trends. Third, we focused on the basic effects of APC and individual-level variables in this study; effects from

\section{REFERENCES}

1. Zeng Y, Feng Q, Hesketh T, Christensen K, Vaupel JW. Survival, disabilities in activities of daily living, and physical and cognitive functioning among the oldest-old in China: a cohort study. Lancet. (2017) 389:1619-29. doi: 10.1016/S0140-6736(17)30548-2

2. National Bureau of Statistics of the People's Republic of China. National data. (2021). Available online at: http://data.stats.gov.cn/easyquery.htm? $\mathrm{cn}=$ C01 (accessed July 10, 2021).

3. United Nations, Department of Economic and Social Affairs, Population Division. World Population Prospects 2019. (2019). Available online at: https:// population.un.org/wpp/DataQuery/ (accessed July 10, 2021).

4. Prince M, Wimo A, Guerchet M, Ali G, Wu Y, Prina M. World Alzheimer Report 2015, the Global Impact of Dementia: An Analysis of Prevalence, Incidence, Cost and Trends. (2015). London: Alzheimer's Disease International (ADI).

5. Wu C, Gao L, Chen S, Dong H. Care services for elderly people with dementia in rural China: a case study. Bull World Health Organ. (2016) 94:167-73. doi: 10.2471/BLT.15.160929 macroeconomic and medical variables on cognitive function should be further explored.

In conclusion, we assessed the trends of cognitive function among the Chinese elderly using the APC model. The gender gaps in cognitive function increased with age and period but decreased with cohorts. The significance of APC effects in shaping social inequalities in cognitive function implied the relevance of both biological forces and historical context. These findings might help inform healthcare planning and priorities for medical resource allocation accordingly.

\section{DATA AVAILABILITY STATEMENT}

Publicly available datasets were analyzed in this study. This data can be found here: https://sites.duke.edu/centerforaging/ programs/chinese-longitudinal-healthy-longevity-survey-clhls/.

\section{ETHICS STATEMENT}

The studies involving human participants were reviewed and approved by the Research Ethics Committees of Duke University and Peking University. The patients/participants provided their written informed consent to participate in this study.

\section{AUTHOR CONTRIBUTIONS}

XH, SG, XZ, XS, YG, and HD contributed to study conception and design. Material preparation and analysis were performed by $\mathrm{XH}, \mathrm{SG}, \mathrm{XZ}, \mathrm{XS}$, and YG. The first draft of the manuscript was written by XH. All authors commented on previous versions of the article. All authors contributed to the article and approved the submitted version.

\section{FUNDING}

This study was funded by the National Natural Science Foundation of China (Grant No.: 71490732).
6. Hu X, Gu S, Sun X, Gu Y, Zhen X, Li Y, et al. Cognitive ageing trajectories and mortality of Chinese oldest-old. Arch Gerontol Geriatr. (2019) 82:81-7. doi: 10.1016/j.archger.2019.01.018

7. Zhou Z, Fu J, Hong YA, Wang P, Fang Y. Association between exercise and the risk of dementia: results from a nationwide longitudinal study in China. BMJ Open. (2017) 7:e17497. doi: 10.1136/bmjopen-2017-017497

8. Finkel D, Reynolds CA, McArdle JJ, Pedersen NL. Cohort differences in trajectories of cognitive aging. J Gerontol B Psychol Sci Soc Sci. (2007) 62:28694. doi: 10.1093/geronb/62.5.p286

9. Sacuiu S, Gustafson D, Sjogren M, Guo X, Ostling S, Johansson B, et al. Secular changes in cognitive predictors of dementia and mortality in 70-year-olds. Neurology. (2010) 75:779-85. doi: 10.1212/WNL.0b013e3181f0737c

10. Christensen K, Thinggaard M, Oksuzyan A, Steenstrup T, Andersen-Ranberg $\mathrm{K}$, Jeune B, et al. Physical and cognitive functioning of people older than 90 years: a comparison of two Danish cohorts born 10 years apart. Lancet. (2013) 382:1507-13. doi: 10.1016/S0140-6736(13)60777-1

11. Steiber N. Population aging at cross-roads: diverging secular trends in average cognitive functioning and physical health in the older population of Germany. PLoS ONE. (2015) 10:e136583. doi: 10.1371/journal.pone.0136583 
12. Gerstorf D, Ram N, Hoppmann C, Willis SL, Schaie KW. Cohort differences in cognitive aging and terminal decline in the Seattle Longitudinal Study. Dev Psychol. (2011) 47:1026-41. doi: 10.1037/a0023426

13. Dodge HH, Zhu J, Lee CW, Chang CC, Ganguli M. Cohort effects in ageassociated cognitive trajectories. J Gerontol A Biol Sci Med Sci. (2014) 69:68794. doi: 10.1093/gerona/glt181

14. Sheffield KM, Peek MK. Changes in the prevalence of cognitive impairment among older Americans, 1993-2004: overall trends and differences by race/ethnicity. Am J Epidemiol. (2011) 174:274-83. doi: 10.1093/aje/kwr074

15. Gao M, Kuang W, Qiu P, Wang H, Lv X, Yang M. The time trends of cognitive impairment incidence among older Chinese people in the community: based on the CLHLS cohorts from 1998 to 2014. Age Ageing. (2017) 46:787-93. doi: 10.1093/ageing/afx038

16. Parker MG, Ahacic K, Thorslund M. Health changes among Swedish oldest old: prevalence rates from 1992 and 2002 show increasing health problems. $J$ Gerontol A Biol Sci Med Sci. (2005) 60:1351-5. doi: 10.1093/gerona/60.10.1351

17. Reither EN, Hauser RM, Yang Y. Do birth cohorts matter? Age-period-cohort analyses of the obesity epidemic in the United States. Soc Sci Med. (2009) 69:1439-48. doi: 10.1016/j.socscimed.2009.08.040

18. Yang Y, Land K. Age-Period-Cohort Analysis: New Models, Methods, and Empirical Applications. (2009). Boca Raton: CRC Press.

19. Yang Y. Trends in U.S. adult chronic disease mortality, 1960-1999: age, period, and cohort variations. Demography. (2008) 45:387-416. doi: $10.1353 /$ dem. 0.0000

20. Zhang L. An age-period-cohort analysis of religious involvement and adult self-rated health: results from the USA, 1972-2008. J Relig Health. (2017) 56:916-45. doi: 10.1007/s10943-016-0292-x

21. Canizares M, Badley EM. Generational differences in patterns of physical activities over time in the Canadian population: an age-period-cohort analysis. BMC Public Health. (2018) 18:304. doi: 10.1186/s12889-018-5189-z

22. Zhang PD, Lv YB, Li ZH, Yin ZX, Li FR, Wang JN, et al. Age, period, and cohort effects on activities of daily living, physical performance, and cognitive functioning impairment among the oldest-old in China. J Gerontol A Biol Sci Med Sci. (2020) 75:1214-21. doi: 10.1093/gerona/glz196

23. Ryder NB. The cohort as a concept in the study of social change. Am Sociol Rev. (1965) 30:843-61. doi: 10.2307/2090964

24. Yang Y. Social inequalities in happiness in the United States, 1972 to 2004: an age-period-cohort analysis. Am Sociol Rev. (2008) 73:204-26. doi: 10.1177/000312240807300202

25. Wu YT, Lee HY, Norton S, Prina AM, Fleming J, Matthews FE, et al. Period, birth cohort and prevalence of dementia in mainland China, Hong Kong and Taiwan: a meta-analysis. Int J Geriatr Psychiatry. (2014) 29:1212-20. doi: $10.1002 /$ gps.4148

26. Zhang Z. Gender differentials in cognitive impairment and decline of the oldest old in China. J Gerontol B Psychol Sci Soc Sci. (2006) 61:S107-15. doi: 10.1093/geronb/61.2.s107

27. Lin SF, Beck AN, Finch BK. Black-white disparity in disability among US older adults: age, period, and cohort trends. J Gerontol B Psychol Sci Soc Sci. (2014) 69:784-97. doi: 10.1093/geronb/gbu010

28. Zeng Y, Vaupel J. Functional capacity and self-evaluation of health and life of oldest old in China. J Soc Issues. (2002) 58:733-48. doi: 10.1111/1540-4560.00287

29. Gu D. General Data Quality Assessment of the CLHLS. In: Zeng Y, Poston D, Vlosky D, Gu D, editors. Healthy Longevity in China: Demographic, Socioeconomic and Psychological Dimensions. (2008). Dordrecht: Springer Netherlands.

30. Folstein MF, Folstein SE, McHugh PR. "Mini-mental state. A practical method for grading the cognitive state of patients for the clinician. J Psychiatr Res. (1975) 12:189.
31. Han L, Gill TM, Jones BL, Allore HG. Cognitive aging trajectories and burdens of disability, hospitalization and nursing home admission among community-living older persons. J Gerontol A Biol Sci Med Sci. (2016) 71:76671. doi: $10.1093 /$ gerona/glv159

32. Lin SF, Beck AN, Finch BK, Hummer RA, Masters RK. Trends in US older adult disability: exploring age, period, and cohort effects. Am J Public Health. (2012) 102:2157-63. doi: 10.2105/AJPH.2011.300602

33. Zhang Z, Gu D, Hayward MD. Early life influences on cognitive impairment among oldest old Chinese. J Gerontol B Psychol Sci Soc Sci. (2008) 63:S25-33. doi: 10.1093/geronb/63.1.s25

34. Raferty A. Choosing models for cross-classifications. Am Sociol Rev. (1986) 1986:145-6.

35. Li T. The New Methodologies of Elderly Health Studies. (2015). Beijing: China Population Publishing House.

36. Chen TY, Chang HY. Developmental patterns of cognitive function and associated factors among the elderly in Taiwan. Sci Rep. (2016) 6:33486. doi: $10.1038 /$ srep33486

37. Lee J, Shih R, Feeney K, Langa KM. Gender disparity in late-life cognitive functioning in India: findings from the longitudinal aging study in India. J Gerontol B Psychol Sci Soc Sci. (2014) 69:603-11. doi: 10.1093/geronb/ gbu017

38. Hayden KM, Reed BR, Manly JJ, Tommet D, Pietrzak RH, Chelune GJ, et al. Cognitive decline in the elderly: an analysis of population heterogeneity. Age Ageing. (2011) 40:684-9. doi: 10.1093/ageing/ afr101

39. Zeng Y, Gu D, Land KC. The association of childhood socioeconomic conditions with healthy longevity at the oldest-old ages in China. Demography. (2007) 44:497-518. doi: 10.1353/dem.2007.0033

40. Ferraro KF, Kelley-Moore JA. Cumulative disadvantage and health: long-term consequences of obesity? Am Sociol Rev. (2003) 68:707-29. doi: $10.2307 / 1519759$

41. Cao J. Longitudinal Changes in Cognitive Function of Old Adults and Its SocioEconomic Differences-Multiple Birth Cohorts Study Based on SDH (Master's thesis) (2019). Nanjing: Nanjing Medical University.

42. Angrisani M, Lee J, Meijer E. The gender gap in education and late-life cognition: Evidence from multiple countries and birth cohorts. J Econ Ageing. (2020) 16:232. doi: 10.1016/j.jeoa.2019.100232

43. Stern Y. What is cognitive reserve? Theory and research application of the reserve concept. J Int Neuropsychol Soc. (2002) 8:448-60. doi: $10.1017 / \mathrm{S} 1355617702813248$

Conflict of Interest: The authors declare that the research was conducted in the absence of any commercial or financial relationships that could be construed as a potential conflict of interest.

Publisher's Note: All claims expressed in this article are solely those of the authors and do not necessarily represent those of their affiliated organizations, or those of the publisher, the editors and the reviewers. Any product that may be evaluated in this article, or claim that may be made by its manufacturer, is not guaranteed or endorsed by the publisher.

Copyright (c) $2021 \mathrm{Hu}, \mathrm{Gu}$, Zhen, Sun, Gu and Dong. This is an open-access article distributed under the terms of the Creative Commons Attribution License (CC BY). The use, distribution or reproduction in other forums is permitted, provided the original author(s) and the copyright owner(s) are credited and that the original publication in this journal is cited, in accordance with accepted academic practice. No use, distribution or reproduction is permitted which does not comply with these terms. 